\title{
RESOLUBILIDADE DO PROCESSO DE RASTREAMENTO DO CÂNCER DE PRÓSTATA NA ATENÇÃo BÁSICA À SAÚdE
}

\author{
Erick de Carvalho Machado ${ }^{1}$, Dayliz Quinto Pereira ${ }^{2}$ \\ 1. Bolsista PIBIC/FAPESB, Graduando em Medicina, Universidade Estadual de Feira de Santana, e-mail: \\ erickmachado.ba@gmail.com \\ 2. Orientador, Departamento de Saúde, Universidade Estadual de Feira de Santana, e-mail: daylizquinto@ @otmail.com
} INTRODUÇÃ̂:

O câncer de próstata é a neoplasia mais frequente entre os homens e quarta causa de morte por neoplasia no Brasil. A incidência aumenta exponencialmente após os 50 anos de idade. Devido à importância desta doença, o Ministério da Saúde criou, em 2001, o Programa Nacional de Controle do Câncer de Próstata que tinha como objetivo diminuir a morbimortalidade associada a esta doença através do rastreamento e detecção precoce (BRASIL, 2002).

As recomendações das sociedades internacionais não são consistentes. O National Cancer Institute não recomenda o rastreamento para população geral, questionando se há redução de mortalidade com a detecção precoce. Enquanto que o American Cancer Society (ACS) sugere que o processo de triagem na população geral inicie a partir dos 50 anos e naqueles pacientes de alto risco, aos 45 anos (INCA, 2013; BRASIL, 2002). Por outro lado, o Ministério da Saúde (2016) orienta a realização do exame de próstata a partir dos 40 anos, sendo gratuito na rede SUS e assegurado pela lei no 10.289, de 20 de setembro de 2001 (Martins et al, 2006; BRASIL, 2016).

Este estudo, portanto, tem como objetivo geral Analisar o processo de triagem e diagnóstico do câncer de próstata sintomático e assintomático na Estratégia de Saúde da Família (ESF), sendo os objetivos específicos: (1) Identificar desafios e perspectivas no processo de triagem e diagnóstico do paciente com câncer de próstata na ESF; (2) Analisar o processo de triagem e diagnóstico do paciente com câncer de próstata; e (3) Conhecer como ocorre a articulação entre a ESF e os serviços da rede de saúde frente à situação de câncer de próstata.

\section{METODOLOGIA:}

Trata-se de um estudo qualitativo. O campo de estudo será a cidade de Feira de SantanaBA, tomando como recorte as ESF onde são oferecidos a comunidade o processo de rastreamento de câncer de próstata. Os participantes do estudo são os médicos que atuam em ESF onde possui um protocolo instituido de triagem e diagnóstico de câncer de próstata. Os critérios de exclusão dos sujeitos nesta pesquisa incluem aqueles profissionais com menos de 1 mês no serviço ou que rejeitarem participar do estudo. A técnica de coleta de dados foi através de entrevista semiestruturada. A análise dos dados foi realizada através da metodologia de análise de conteúdo, e para isso as entrevistas serão gravadas na íntegra. Segundo Bardin (apud MINAYO; 
DESLANDES; GOMES, 2007), a análise do conteúdo é um conjunto de técnicas de análise das comunicações, tudo isso com o intuito de obter indicadores que permitam a inferência de conhecimentos relativos às condições de produção/percepção destas mensagens. Esta pesquisa foi aprovada pelo CEP local, conforme o CAAE: 61079316.0.0000.0053.

\section{RESULTADOS E DISCUSSÕES:}

\subsection{Categoria Temática 1 - Adesão do público masculino aos programas de saúde de Atenção} à Saúde do Homem:

Diante da ideia de invisibilidade masculina na Atenção à Saúde decorrente a escassez de programas centrados na saúde desta população, foi objetivado pelo Ministério da Saúde, em 2009, a criação do Programa Nacional de Atenção Integral à Saúde do Homem (PNAISH), que possui como princípio básico a orientação de ações e serviços de saúde para população de homens entre 20 e 59 anos, com garantia de integralidade, equidade e humanização do atendimento (Leal et al, 2012; Moura et al, 2012). Entretanto, as ABS, em geral, não possuem serviços de saúde centrados na saúde do homem, resumindo suas ações às palestras sobre hipertensão e diabetes.

A baixa adesão dos homens aos programas de saúde é outro fator que contribui para maior mortalidade e associa-se ao processo de socialização masculino com desvalorização do auto cuidado, invulnerabilidade e ao comportamento de risco. Os fatores relacionados ao serviço de saúde incluem tempo perdido na espera da assistência e por considerarem um espaço feminilizado, frequentado principalmente por mulheres e composto por uma equipe de profissionais formada, em sua maioria, também por mulheres.

\subsection{Categoria Temática 2 - Resolubilidade do rastreamento de câncer de próstata na ABS:}

Não existe um consenso entre as entidades médicas sobre a realização do rastreamento na população geral e qual a idade ideal para início do rastreamento (Martins et al, 2006; INCA, 2013). No Brasil, o Ministério da Saúde (2016) orienta a realização do exame de próstata a partir dos 40 anos, devendo ser realizado anualmente (Martins et al, 2006; BRASIL, 2016). Dentre os procedimentos para o rastreamento pode ser realizado por quantificação de PSA sérico e toque retal (Martins et al, 2006). No nosso estudo, os médicos admitiram realizar somente a avaliação do PSA sérico, justificando a restrição dos pacientes e falta de habilidade na avaliação do toque retal.

Devido à realização somente do PSA sérico, há um aumento dos falsos-positivos o que sobrecarrega a rede de serviços devido ao sistema de cotas. Este fato reflete-se no tempo de espera 
para realização de consulta com o urologista que variou de 20 a 60 dias no nosso estudo. Em paralelo, Nascimento e cols (2015) referiu que o tempo médio de espera para realização da primeira consulta após colposcopia alterada em mulheres era de 20 a 60 dias, enquanto que Nascimento e Silva (2015) evidenciou que as mulheres iniciaram a radioterapia dentro de 60 dias da confirmação diagnóstica. Apesar do tempo ser condizente com o que é encontrado na literatura em relação as mulheres, esse intervalo pode causar um retardo no diagnóstico do câncer de próstata e, por conseguinte, seu tratamento.

\subsubsection{Categoria Temática 3-Articulação da rede de saúde frente o rastreamento de câncer de próstata:}

Frente as dificuldades no rastreamento de câncer de próstata, é esperado uma atuação ativa da Secretária Municipal de Saúde (SMS) na realização de atividades de atualização e programas de saúde voltadas para públicos específicos. Contudo, há uma atuação tímida da SMS, sobretudo na atualização destes profissionais.

Confronta-se com estas informações, a dificuldade dos profissionais entrevistados na realização do toque retal. Em detrimento a isto, o Plano Nacional de Atenção Básica (2012) defende a educação permanente com base em cursos de atualização periódicos até o aprendizado que parte dos problemas e desafios enfrentados no processo de trabalho (BRASIL, 2012).

\section{CONSIDERAÇÕES FINAIS:}

O rastreamento de câncer de próstata ainda é um descenso na literatura. No Brasil, o Ministério da Saúde orienta para realização de um programa de triagem para diagnóstico precoce de câncer de próstata. Apesar disto, os profissionais médicos da ABS não realizam o com dificuldade e de forma não protocolada. Nesse sentido, é imprescindível a atuação de gestores na promoção de cursos de atualização e na fiscalização do processo de rastreamento.

\section{REFERENCIAS:}

1- ACS. American Cancer Society. Prostate Cancer: Early Detection. Disponível em: <http://www.cancer.org/acs/groups/cid/documents/webcontent/003182-pdf.pdf>. Acesso em: 27 mar 2016

2- ARAÚJO, EM et al. Mortalidade masculina no estado da Bahia, regiões Nordeste e Sudeste do Brasil no período de 2000 a 2009. Saúde do Homem no SUS. Vol 14/1. Ago 2012. Disponível em: < http://portal.saude.sp.gov.br/resources/instituto-de--saude/homepage/bis/pdfs/bis_v14_1.pdf>. Acesso em: 27 mar 2016 
3- BACELAR JÚNIOR, AJ et al. Câncer de próstata: métodos de diagnóstico, prevenção e tratamento. Braz. J. Surg. Clin. Res. V.10,n.3,pp.40-46 (Mar - Mai 2015) Disponível em: < http://www.mastereditora.com.br/periodico/20150501_174533.pdf> Acessado em: 04 jun 2016

4- BARDIN, L. Análise de conteúdo. Lisboa, Portugal; Edições 70, LDA, 2009.

5- BRASIL, Ministério da Saúde, Saúde do Homem. Disponível em: <http:/bvsms.saude.gov.br/bvs/legislacao/homem.php>. Acesso em: 27 mar 2016

6- BRASIL. Ministério da Saúde. Secretaria de Assistência à Saúde. Instituto Nacional de Câncer. Programa nacional de controle do câncer da próstata: documento de consenso. - Rio de Janeiro: INCA, 2002.

7- BRASIL. Ministério da Saúde. Secretaria de Atenção à Saúde. Departamento de Atenção Básica. Política Nacional de Atenção Básica. Brasilia: Ministério da Saúde, 2012.

8- FIGUEIREDO W. Assistência à saúde dos homens: um desafio para os serviços de atenção primária. Ciencia \& Saúde Coletiva, 10(1):105-109, 2005

9- GOMES R, NASCIMENTO EF, ARAÚJO FC. Porque os homens buscam menos os serviços de saúde do que as mulheres? As explicações de homens com baixa escolaridade e homens com ensino superior. Cad. Saúde Pública, Rio de Janeiro, 23(3): 565-574, 2007

10- INCA, Instituto Nacional de Câncer. Rastreamento do Câncer de Próstata. 2013. Disponível em: $<$ http://www1.inca.gov.br/inca/Arquivos/rastreamento_prostata_resumido.2013.pdf $>$. Acesso em: 27 mar 2016

11-LEAL, AF; FIGUEIREDO, WS, SILVA, GSN da. O percusso da Política Nacional de Atenção Integral à Saúde dos Homens, desde sua formulação até sua implementação nos serviços públicos locais de atenção à saúde. Ciência \& Saúde Coletiva, 17 (10): 2607-2616, 2012.

12- MARTINS, ACP et al. Câncer de Próstata: Prevenção e Rastreamento. Projeto Diretrizes. 2006 jun. Disponível em: <http://projetodiretrizes.org.br/5_volume/10-CancerPrev.pdf> Acesso em: 02 de jun de 2016

13- MOTTET, $\mathrm{N}$ et al. EAU guidelines on prostate cancer. Eur Urol 2015 Março; Disponível em: <http://uroweb.org/wp-content/uploads/09-Prostate-Cancer_LR.pdf> Acesso em: 02 de jun de 2016

14- MOURA EC, LIMA AMP, URDANETA M. Uso de indicadores para o monitoramento das ações de promoção e atenção da Política Nacional de Atenção Integral à Saúde do Homem. Ciência \& Saúde Coletiva, 17(10): 2597-2606, 2012

15- MOURA E. Perfil da Situação de Saúde do Homem no Brasil. Rio de Janeiro: Fundação Oswaldo Cruz, 2012

16-STEVENS A, SCHMIDT MI, DUNCAN BB. Desigualdades de gênero na mortalidade por doenças crônicas não transmissíveis no Brasil. Ciência \& Saúde coletiva 17(10): 2627-2634, 2012

17-SOUTO, CAV et al. Câncer de Próstata: Marcadores Tumorais. Projeto Diretrizes. 2006 jun. Disponível em: 〈http//www.projetodiretrizes.org.br/5_volume/09-CancerMar.pdf〉 Acesso em: 02 de jun de 2016

18- VIEIRA, CG; ARAÚJO, W de S; VARGAS, DRM de. O homem e o câncer de próstata: prováveis reações diante de um possível diagnóstico. Revista Científica do ITPAC, Araguaína, v.5, n.1, Pub.3, Janeiro 2012 